\title{
Um estudo empírico de um sistema de reconhecimento facial utilizando o classificador KNN
}

\author{
Fabio Abrantes Diniz ${ }^{1}$ \\ Thiago Reis da Silva ${ }^{2}$ \\ Francisco Eduardo Silva Alencar ${ }^{3}$
}

\begin{abstract}
Resumo: Neste artigo, apresenta-se um estudo empírico de otimização das taxas de acurácias resultantes de um sistema de reconhecimento facial baseado nas técnicas Eigenfaces e K-Nearest Neighbors. Foram investigadas as seguintes variáveis: imagens com três dimensões distintas, número de características (Eigenfaces), valores de $k$ da técnica K-Nearest Neighbors e três medidas de distância (euclidiana, Manhattan e euclidiana normalizada). Os estudos foram importantes para entender empiricamente quais parâmetros são os mais relevantes para as técnicas analisadas e que resultam em melhores taxas de acurácias de reconhecimento facial. Os resultados dos experimentos comprovaram que as imagens com dimensões 12x9 pixels produzem as melhores taxas de acurácias de reconhecimento facial, combinando com a medida de distância euclidiana normalizada e um número de Eigenfaces igual a vinte.
\end{abstract}

Palavras-chave: Estudo empírico. Reconhecimento facial. Eigenfaces. K-Nearest Neighbors.

\begin{abstract}
In this article, we present an empirical study of optimization of accuracies rates resulting from a facial recognition system based on technical Eigenfaces and K-Nearest Neighbors. The following variables were investigated: images with three different dimensions, number of features (Eigenfaces), $k$ values of the K-Nearest Neighbors and technical three distance measurements (Euclidean, Manhattan and the normalized Euclidean). The studies were important to understand, empirically, what are the most relevant parameters for the analyzed techniques that result the best accuracies rates of facial recognition. The results of the experiments showed that the images with dimensions $12 \times 9$ produce the best rates accuracies of facial recognition, matching the standard measure of Euclidean distance and a number of Eigenfaces equal to twenty.
\end{abstract}

Keywords: Empirical study. Face Recognition. Eigenfaces. K-Nearest Neighbors.

\section{Introdução}

O reconhecimento facial é um dos processos de identificação mais utilizado pelos seres humanos, pois permite identificar rapidamente qualquer indivíduo. Embora o reconhecimento facial seja uma tarefa simples para o ser humano, não é trivial implementar esse processo em uma máquina. A grande dificuldade está na modelagem de uma face que abstraia as características que as diferenciem de outras faces, já que apresentam poucas diferenças substanciais entre si [1]. Pois, embora diferentes, todas as faces possuem características semelhantes, uma boca, dois olhos e um nariz. Além disso, imagens com diferentes dimensões dificultam o processo de reconhecimento facial, principalmente nas etapas de extração das características faciais e

\footnotetext{
${ }^{1}$ Instituto Federal de Educação, Ciência e Tecnologia do Piauí - IFPI, campus Paulistana.

\{fabio.abrantes.dinizeifpi.edu.br\}

${ }^{2}$ Instituto Federal de Educação, Ciência e Tecnologia do Maranhão - IFMA, campus São Raimundo das Mangabeiras. Doutorando em Sistemas e Computação na Universidade Federal do Rio Grande do Norte - UFRN.

\{thiago.reiseifma.edu.br\}

${ }^{3}$ Universidade do Estado do Rio Grande do Norte, Universidade Federal Rural do Semi-Árido - UFERSA.

\{eduardu.dudu@gmail.com\}
}

http://dx.doi.org/10.5335/rbca.2015.5227

Revista Brasileira de Computação Aplicada (ISSN 2176-6649), Passo Fundo, v. 8, n. 1, p. 50-63, abr. 201650 
classificação, pois quanto maior é a dimensionalidade da imagem, maior é a matriz de vetores e, portanto, maior é o custo computacional e menor é a precisão do classificador.

Vários algoritmos de reconhecimento facial foram propostos [2] a fim de resolver esses problemas. No presente trabalho é apresentado um estudo empírico de otimização dos resultados da variável dependente taxa de acurácia de um sistema de reconhecimento facial [3] baseado nas técnicas de Eigenfaces [4] e K-Nearest Neighbors (K-NN) [5]. Foram analisadas as seguintes variáveis independentes das técnicas abordadas:

a) três dimensões das imagens;

b) número de características faciais (Eigenfaces);

c) o valor de $k$ da técnica K-NN;

d) o uso de três medidas de distâncias (euclidiana, Manhattan e euclidiana normalizada).

Este estudo preocupou-se com a análise dos problemas de dimensionalidade de imagens para o reconhecimento facial ao verificar quais dimensões de imagens são ideais para fornecer as características faciais mais significativas, além disso, proporcionou uma análise dos valores dos parâmetros para as técnicas abordadas.

O presente estudo foi realizado por meio de um experimento que utilizou uma base de dados própria contendo 1.280 imagens de um total de 64 indivíduos. Cada indivíduo dessa base de dados foi representado por vinte imagens em cinco poses distintas. De acordo com os principais resultados dos testes do experimento, verificou-se que imagens de dimensões diferentes produziram taxas de acurácias diferentes. Além disso, evidenciou-se que a melhor acurácia no sistema reconhecimento facial foi encontrada na seguinte combinação de parâmetros: dimensão $12 \times 9$ pixels, distância euclidiana normalizada, valor de $k$ igual a um e número de característica igual a vinte. Comprovou-se, também, que as imagens de menor dimensão analisadas (12x9) produziram as melhores taxas de acurácias de reconhecimento facial em relação às outras dimensões estudadas.

Portanto, este artigo segue organizado em sete seções, a partir desta introdução. A seção 2 descreve o sistema de reconhecimento facial abordado e suas técnicas, a seção 3 apresenta o planejamento do experimento, e suas subseções, as questões de pesquisa e hipóteses (3.1), as definições formais das hipóteses (3.2), as variáveis dependentes e independentes (3.3), o design do experimento e as unidades experimentais (3.4). Na seção 4, são apresentados os resultados do estudo, nas suas subseções são expostas as identificações do modelo matemático (4.1) e a validação do modelo (4.2). Na seção 5 , descreve-se a análise de variância, nas suas subseções, a alocação de variação (5.1), a significância dos efeitos (5.2) e a verificação das hipóteses (5.3). Posteriormente, na seção 6, são apresentadas as discussões das verificações das hipóteses, na seção 7, as ameaças à validade do experimento, e, por fim, a seção 8 apresenta as conclusões e indicações para trabalhos futuros.

\section{Sistema de reconhecimento facial abordado}

O sistema de reconhecimento facial proposto por Diniz et al. [3] é baseado na técnica de Eigenfaces e no classificador K-NN. A arquitetura do sistema de reconhecimento facial consiste-se basicamente nas seguintes etapas: a) aquisição das imagens, b) pré-processamento, c) extração de características, d) classificação, e) verificação.

O início do processo de reconhecimento de face dá-se a partir do módulo de aquisição da imagem. Nas simulações deste trabalho, foi utilizada uma base de dados própria, com um total de 64 indivíduos. Cada indivíduo integrante da base de dados é representado por vinte imagens nas seguintes posições: quatro imagens com o indivíduo olhando diretamente para a câmera, quatro, olhando 45 graus à esquerda da câmera, quatro, olhando 45 graus à direita da câmera, quatro olhando 90 graus à esquerda da câmera e nas últimas quatro imagens, o indivíduo olha 90 graus à direita da câmera, totalizando 1.280 imagens. Todas as imagens foram obtidas em um ambiente homogêneo e com iluminação controlada (foi utilizado o mesmo cenário em todas as imagens).

$\mathrm{Na}$ etapa de pré-processamento, o sistema detectou a imagem da face do usuário utilizando implementação da técnica de detecção de face, como proposto por Viola e Jones [6]. Em seguida, as faces capturadas tiveram correções nas suas dimensões (todas foram redimensionadas para os tamanhos em 120x90

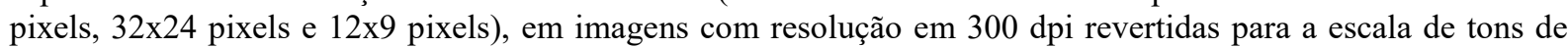
cinza.

Revista Brasileira de Computação Aplicada (ISSN 2176-6649), Passo Fundo, v. 8, n. 1, p. 50-63, abr. 201651 
Após o pré-processamento, a imagem da face normalizada serviu de entrada para o módulo de extração de características, com o objetivo de encontrar as principais características a serem utilizadas para a classificação. Cada imagem do indivíduo foi transformada em uma matriz de tamanho $\mathrm{w} \times \mathrm{h}$, em que $\mathrm{w}$ e $\mathrm{h}$ são, respectivamente, os números de pixels referentes à largura e à altura da imagem. Cada valor do pixel da imagem corresponde a uma componente do vetor.

Devido à alta dimensão dos vetores, nessa etapa, foi implementada a técnica de Eigenfaces, a fim de reduzir a quantidade de características de uma imagem. O algoritmo Eigenface visa fornecer um conjunto de vetores de distribuições probabilísticas para resolver o problema da detecção de padrões em imagens, e seu fundamento básico é a utilização desses vetores para gerar uma informação matemática do rosto de um ser humano para sua futura identificação.

Com a ajuda de um classificador de padrões, as características extraídas da imagem da face são comparadas com as amostras do conjunto de treinamento de face. A imagem da face é então classificada como conhecida ou desconhecida. Neste trabalho, foi analisado o algoritmo de reconhecimento de padrão K-NN [2].

A última etapa da arquitetura é o conjunto de treinamento, que visa encontrar características apropriadas para a representação de padrões de entrada, sendo o classificador treinado para particionar o espaço de características, formando padrões de todas as classes existentes. Foram usadas $75 \%$ das imagens faciais da base de dados para o conjunto de treinamento e $25 \%$ das imagens para os testes. Na seção seguinte, estão detalhadas as técnicas de Eigenfaces e K-NN.

\subsection{Eigenfaces e o classificador K-Nearest Neighbors}

O K-NN é um dos algoritmos de classificação mais utilizados na área de aprendizagem de máquina [3]. É baseado na procura dos $k$ vizinhos mais próximos do padrão de teste. A busca pela vizinhança é feita utilizando uma medida de distância nessa procura. Nos experimentos desenvolvidos neste trabalho, além da medida euclidiana, foram utilizadas as distâncias Manhattan e euclidiana normalizada [3]. A implementação do algoritmo K-NN foi realizada com peso pela distância, e não pela frequência. Dessa maneira, o padrão é classificado de acordo com a soma dos pesos dos $k$ vizinhos - o peso é o inverso das distâncias. O $k$ escolhido será aquele que proporcionar menor quantidade de erros na classificação das imagens [3].

Já o método Eigenfaces busca um conjunto de características que não dependa das formas geométricas da face (olhos, nariz, orelhas e boca) e utiliza toda a informação da representação facial [4]. Baseada na teoria da informação, as Eigenfaces identificam um pequeno número de características que são relevantes para diferenciar uma face de outras faces.

Essas características podem ser analisadas apenas com a variação dos valores assumidos pelos pixels, em um conjunto de imagens de faces. Os autovetores da matriz de covariância de um conjunto de imagens de faces descrevem a variação dos pixels em uma região diferente das imagens, ou seja, cada autovetor descreve a variação dos pixels associados a diferentes características faciais [4].

Depois de encontrar os autovetores, as imagens de faces são projetadas em um espaço de características, chamado Espaço de Faces, que melhor representa a variação entre faces conhecidas. Esse espaço é definido pelas Eigenfaces, que são os autovetores do conjunto de faces. O reconhecimento de uma face é realizado pela sua projeção no subespaço gerado pelas Eigenfaces, realizando a comparação da posição obtida com a posição de indivíduos conhecidos [4].

A implementação da sequência dos procedimentos da geração das Eigenfaces conforme disposto pelo estudo de Cheon e Kim [7]:

a) adquirir um conjunto de $M$ faces. Em que $\Gamma_{i}(i \in N)$ é cada face do conjunto da base de treinamento;

$$
\Gamma=\left[\Gamma_{1}, \Gamma_{2}, \ldots, \Gamma_{M}\right]
$$

b) calcular a face média $\Psi(2)$ para eliminar informações redundantes na face, isto é, uma imagem de face poderia ser representada com poucos componentes principais devido à sua redundância;

$$
\Psi=\frac{\sum_{i=1}^{M} \Gamma_{i}}{M}
$$

Revista Brasileira de Computação Aplicada (ISSN 2176-6649), Passo Fundo, v. 8, n. 1, p. 50-63, abr. 201652 
c) criar uma matriz com as faces de treino com os pixels dispostos em linhas e as $M$ faces do conjunto de treinamento dispostas em colunas;

d) subtrair a imagem média $\Psi$ de cada imagem de $\Gamma$, gerando uma nova matriz $A$ (3), que contém somente as variações de cada face em relação à face média. $\mathrm{O}$ vetor $\Phi(4)$ contém todas as variações de uma determinada face $\Gamma$ em relação à face média $\Psi$;

$$
\begin{aligned}
& A=[\Phi 1, \Phi 2, \ldots, \Phi m] \\
& \Phi=\Gamma-\Psi
\end{aligned}
$$

e) sendo $M$ menor que a dimensionalidade (largura multiplicada pela altura das imagens de treinamento) das imagens em $\Phi$, calcular a matriz de covariância $C$ (5);

$C=A A^{T}$

f) calcular os autovetores $v$ e autovalores $\lambda$ da matriz $C$;

g) criar a matriz de transformação. A matriz $\mu$ (6) conterá $M-1$ autovetores significativos. Entretanto, ainda é possível realizar a eliminação de alguns desses $M-1$ autovetores pela ordem de importância de seus correspondentes autovalores $\lambda$, gerando $M^{\prime}$ autovetores escolhidos;

$\mu=\nu \Phi$

h) normalizar os $M^{\prime}$ vetores da matriz $\mu$;

i) as imagens do conjunto de treinamento são projetadas no espaço de faces, efetuando-se a operação de transformação (6). Em seguida, o novo padrão passa para a fase de classificação (7).

$\Omega=\mu^{T} \Phi$

\section{Planejamento do experimento}

O planejamento deste estudo permitiu o aprimoramento de processos, a redução da variabilidade de resultados, a redução do tempo de análise e dos custos envolvidos. Nesta seção, é detalhada a condução do experimento, seguindo as orientações propostas por Wohlin et al. [8] e Kitchenham, Pickard e Pfleeger [9].

\subsection{Questões de pesquisa e hipóteses}

A métrica acurácia é amplamente utilizada para avaliar a eficácia de um sistema de reconhecimento facial. A eficácia da acurácia do sistema está relacionada à sua capacidade de reconhecer corretamente os indivíduos pela sua face.

Sendo assim, a principal questão de pesquisa (QP) objetiva comparar e determinar os melhores valores dos fatores das técnicas abordadas no sistema de reconhecimento facial a fim de melhorar a acurácia desse sistema quando alimentado com imagens faciais de dimensões diferentes. Baseando-se nessas informações sobre o reconhecimento facial, foram formuladas outras questões:

- QP1: O sistema de reconhecimento facial apresenta resultados diferentes na métrica de acurácia quando temos como entrada imagens faciais com dimensões faciais diferentes?

A questão leva às seguintes hipóteses:

- H1-0: a acurácia do sistema de reconhecimento facial para imagens faciais com dimensões faciais diferentes é igual;

- H1-1: a acurácia do sistema de reconhecimento facial para imagens faciais com dimensões faciais diferentes é diferente.

A fim de que os resultados finais da pesquisa sejam bem compreendidos, é necessário conhecer as interações entre os fatores e a variável resposta. Dessa forma, é preciso saber quais dos fatores do experimento são mais responsáveis pela variação nas saídas obtidas nas execuções dos tratamentos. Para isso, as seguintes questões foram definidas: 
- QP2: Qual distância aplicada na dimensão específica da imagem facial produz melhores taxas de acurácias no reconhecimento facial?

- QP3: Qual número de característica (Eigenfaces) aplicada na dimensão específica da imagem facial produz melhores taxas de acurácias no reconhecimento facial?

- QP4: Qual número de $k$ do classificador K-NN aplicada na dimensão específica da imagem facial produz a melhor taxa de acurácia?

Esses questionamentos levam às seguintes hipóteses:

- H2-0: a acurácia do sistema de reconhecimento facial utilizando distintas distâncias é igual.

- H2-1: a acurácia do sistema de reconhecimento facial utilizando distintas distâncias é diferente.

- H3-0: a acurácia do sistema de reconhecimento facial utilizando distintos valores de $k$ é igual.

- H3-1: a acurácia do sistema de reconhecimento facial utilizando distintos valores de $k$ é diferente.

- H4-0: a acurácia do sistema de reconhecimento facial para imagens faciais utilizando distintas Eigenfaces é igual.

- H4-1: a acurácia do sistema de reconhecimento facial para imagens faciais utilizando distintas Eigenfaces é diferente.

\subsection{Definições formais das hipóteses}

Formalmente, as quatro hipóteses descritas anteriormente estão definidas na Tabela 1. Todas as variáveis - e suas entradas - estão definidas na subseção seguinte.

Tabela 1: Formulações das hipóteses

\begin{tabular}{ccc}
\hline Hipótese & Hipótese nula & Hipótese alternativa \\
\hline $\mathrm{H} 1$ & $\mathrm{H} 1_{0}=\mathrm{A}\left(\operatorname{Dim}_{1}\right)=\mathrm{A}\left(\mathrm{Dim}_{2}\right)=\mathrm{A}$ & $\mathrm{H} 1_{1}=\mathrm{A}\left(\operatorname{Dim}_{1}\right) \neq \mathrm{A}\left(\operatorname{Dim}_{2}\right) \neq \mathrm{A}$ \\
& $\left(\mathrm{Dim}_{3}\right)$ \\
$\mathrm{H} 2$ & $\mathrm{H} 2_{0}=\mathrm{A}\left(\mathrm{D}_{1}\right)=\mathrm{A}\left(\mathrm{D}_{2}\right)=\mathrm{A}\left(\mathrm{D}_{3}\right)$ & $\mathrm{H} 2_{1}=\mathrm{A}\left(\mathrm{D}_{1}\right) \neq \mathrm{A}\left(\mathrm{D}_{2}\right) \neq \mathrm{A}\left(\mathrm{D}_{3}\right)$ \\
$\mathrm{H} 3$ & $\mathrm{H} 3_{0}=\mathrm{A}\left(\mathrm{k}_{1}\right)=\mathrm{A}\left(\mathrm{k}_{3}\right)=\mathrm{A}\left(\mathrm{k}_{4}\right)=\mathrm{A}$ & $\mathrm{H} 3_{1}=\mathrm{A}\left(\mathrm{k}_{1}\right) \neq \mathrm{A}\left(\mathrm{k}_{3}\right) \neq \mathrm{A}\left(\mathrm{k}_{4}\right)$ \\
& $\left(\mathrm{k}_{5}\right)=\mathrm{A}\left(\mathrm{k}_{6}\right)=\mathrm{A}\left(\mathrm{k}_{7}\right)=\mathrm{A}\left(\mathrm{k}_{8}\right)=\mathrm{A}$ & $\neq \mathrm{A}\left(\mathrm{k}_{5}\right) \neq \mathrm{A}\left(\mathrm{k}_{6}\right) \neq \mathrm{A}\left(\mathrm{k}_{7}\right) \neq \mathrm{A}$ \\
$\left(\mathrm{k}_{9}\right)=\mathrm{A}\left(\mathrm{k}_{10}\right)$ & $\left(\mathrm{k}_{8}\right) \neq \mathrm{A}\left(\mathrm{k}_{9}\right) \neq \mathrm{A}\left(\mathrm{k}_{10}\right)$ \\
& & \\
& & \\
$\mathrm{H} 4$ & $\mathrm{H} 4_{0}=\mathrm{A}\left(\mathrm{E}_{15}\right)=\mathrm{A}\left(\mathrm{E}_{16}\right)=\mathrm{A}\left(\mathrm{E}_{17}\right)=$ & $\mathrm{H} 41=\mathrm{A}\left(\mathrm{E}_{15}\right) \neq \mathrm{A}\left(\mathrm{E}_{16}\right) \neq \mathrm{A}$ \\
& $\mathrm{A}\left(\mathrm{E}_{18}\right)=\mathrm{A}\left(\mathrm{E}_{19}\right)=\mathrm{A}\left(\mathrm{E}_{20}\right)$ & $\left(\mathrm{E}_{17}\right) \neq \mathrm{A}\left(\mathrm{E}_{18}\right) \neq \mathrm{A}\left(\mathrm{E}_{19}\right) \neq \mathrm{A}$ \\
& & $\left(\mathrm{E}_{20}\right)$
\end{tabular}

A é a função que retorna o valor da métrica acurácia aplicada nas seguintes variáveis: dimensões $\left(\mathbf{D i m}_{1}, \mathbf{D i m}_{2} \mathrm{e} \mathbf{D i m}_{3}\right)$, distâncias $\left(\mathbf{D}_{1}, \mathbf{D}_{2}\right.$ e $\left.\mathbf{D}_{3}\right)$, Eigenfaces $\left(\mathbf{E}_{15}, \mathbf{E}_{16}, \mathbf{E}_{17}, \mathbf{E}_{18}, \mathbf{E}_{19}\right.$ e $\left.\mathbf{E}_{20}\right)$, valor de $k\left(\mathbf{A}\left(\mathbf{k}_{1}\right)=\mathbf{A}\left(\mathbf{k}_{3}\right)=\mathbf{A}\left(\mathbf{k}_{4}\right)=\mathbf{A}\left(\mathbf{k}_{5}\right)=\mathbf{A}\left(\mathbf{k}_{6}\right)=\mathbf{A}\left(\mathbf{k}_{7}\right)=\mathbf{A}\left(\mathbf{k}_{8}\right)=\mathbf{A}\left(\mathbf{k}_{9}\right)=\mathbf{A}\left(\mathbf{k}_{10}\right)\right)$.

Fonte: elaboração dos autores com base nos dados da pesquisa.

\subsection{Variáveis independentes e dependentes}

As variáveis independentes (também chamadas de fatores) são todas aquelas que podem ser manipuladas ou controladas no processo de experimentação, enquanto as variáveis dependentes são apenas medidas dependentes da manipulação ou das condições experimentais [10]. Assim, descreve-se, a seguir, a etapa que selecionaram as variáveis independentes (fatores de controles) com suas respectivas faixas de variação de níveis e as variáveis dependentes (variáveis-respostas). Os níveis dos fatores estão definidos de acordo com a Tabela 2. 
Tabela 2: Níveis dos fatores

\begin{tabular}{ccc}
\hline Fator & Tipo de Variável & Nível \\
\hline Dimensão das imagens faciais & Qualitativa & $120 \times 90,32 \times 24,12 \times 9$ \\
Eigenfaces & Quantitativa & $15,16,17,18,19,20$ \\
Vizinhos mais próximos $(k)$ & Quantitativa & $1,3,4,5,6,7,8,9,10$ \\
Distâncias & Qualitativa & euclidiana, Manhattan e \\
& & euclidiana normalizada \\
\hline Fonte: elaboração dos autores com base nos dados da pesquisa
\end{tabular}

Fonte: elaboração dos autores com base nos dados da pesquisa.

No caso deste estudo, é analisada somente a taxa de acurácia como a variável dependente. Já as variáveis independentes utilizadas no experimento são:

a) dimensão das imagens faciais: cada imagem representante do indivíduo foi transformada em uma matriz de tamanho $\mathrm{w} x \mathrm{~h}$, em que w e h são, respectivamente, os números de pixels referentes à largura e à altura da imagem;

b) características principais (Eigenfaces): conjunto de vetores de distribuições probabilísticas (autovetores da matriz de covariância) que gera uma informação matemática do rosto de um ser humano [4];

c) vizinhos mais próximos $(\boldsymbol{k})$ : o algoritmo é baseado na procura dos $k$ vizinhos mais próximos do padrão de teste. $\mathrm{O}$ padrão é dito pertencer à classe que apresentar a maior frequência dentre os $k$ vizinhos utilizados [5];

d) distâncias: a busca pela vizinhança pelo algoritmo K-NN [5] é feita utilizando uma medida de distância nessa procura.

\subsection{Design do experimento e unidades experimentais}

O design experimental utilizado foi o planejamento fatorial completo com dez replicações. Foram elaborados 486 ensaios, executados dez vezes cada um, totalizando 4.860 execuções. Como cada execução tem custo mínimo, não há problema em usar esse tipo de design. A Tabela 3 descreve cada um dos tratamentos.

Tabela 3: Desenho fatorial completo

\begin{tabular}{ccccccccccc}
\hline \multicolumn{10}{c}{ Dimensões $=\mathbf{1 2 0 x 9 0 , 1 2 \times 9} \mathbf{3 2 x 2 4}$} \\
\hline Distância & Eigenface & K1 & K3 & K4 & K5 & K6 & K7 & K8 & K9 & K10 \\
\hline Euclidiana & 15 & 1 & 3 & 4 & 5 & 6 & 7 & 8 & 9 & 10 \\
& 16 & 1 & 3 & 4 & 5 & 6 & 7 & 8 & 9 & 10 \\
& 17 & 1 & 3 & 4 & 5 & 6 & 7 & 8 & 9 & 10 \\
Euclidiana & 18 & 1 & 3 & 4 & 5 & 6 & 7 & 8 & 9 & 10 \\
normalizada & 19 & 1 & 3 & 4 & 5 & 6 & 7 & 8 & 9 & 10 \\
& 20 & 1 & 3 & 4 & 5 & 6 & 7 & 8 & 9 & 10 \\
& 15 & 1 & 3 & 4 & 5 & 6 & 7 & 8 & 9 & 10 \\
& 16 & 1 & 3 & 4 & 5 & 6 & 7 & 8 & 9 & 10 \\
Manhattan & 17 & 1 & 3 & 4 & 5 & 6 & 7 & 8 & 9 & 10 \\
& 18 & 1 & 3 & 4 & 5 & 6 & 7 & 8 & 9 & 10 \\
& 19 & 1 & 3 & 4 & 5 & 6 & 7 & 8 & 9 & 10 \\
& 20 & 1 & 3 & 4 & 5 & 6 & 7 & 8 & 9 & 10 \\
& 15 & 1 & 3 & 4 & 5 & 6 & 7 & 8 & 9 & 10 \\
& 16 & 1 & 3 & 4 & 5 & 6 & 7 & 8 & 9 & 10 \\
& 17 & 1 & 3 & 4 & 5 & 6 & 7 & 8 & 9 & 10 \\
& 18 & 1 & 3 & 4 & 5 & 6 & 7 & 8 & 9 & 10 \\
& 19 & 1 & 3 & 4 & 5 & 6 & 7 & 8 & 9 & 10 \\
& 20 & 1 & 3 & 4 & 5 & 6 & 7 & 8 & 9 & 10 \\
\hline
\end{tabular}

Neste experimento, as unidades experimentais são os conjuntos de imagens faciais de cada dimensão. Para cada dimensão, serão extraídas, randomicamente, imagens faciais para o conjunto de treinamento e para o conjunto de teste. Logo, cada conjunto de treinamento de sua respectiva dimensão é uma amostra aleatória sob a qual o "tratamento" do experimento (conjunto de fatores) é aplicado para se obter a variável dependente 
abordada. É por meio dessas unidades que será possível obter a variação estatística na análise dos resultados da investigação. Houve uma randomização para a formação da base de treinamento com $75 \%$ das imagens faciais da base de dados utilizadas para o conjunto de treinamento e $25 \%$ das imagens para os testes.

\section{Resultados do experimento}

Esta seção apresenta a análise dos dados coletados durante a execução do experimento. Os resultados coletados se encontram empacotados no respectivo $\operatorname{link}^{4}$. Para a variável dependente abordada, esta análise inclui a análise da identificação do modelo matemático, a validação do modelo matemático e a análise de variação dos efeitos.

\subsection{Identificação do modelo matemático}

O modelo teórico do design escolhido (fatorial completo com quatro fatores e dez repetições) considera quatro efeitos principais $(\alpha, \beta, y, \lambda)$, seis interações entre dois fatores $(\alpha \beta, \alpha y, \alpha \lambda, \beta \lambda, \beta \lambda, y \lambda)$, quatro interações entre três fatores $(\alpha \beta y, \alpha \beta \lambda, \alpha y \lambda, \beta y \lambda)$ e uma interação com quatro fatores $(\alpha \beta y \lambda)$. Esse modelo pode ser expresso pela Equação (8) [11], apresentada a seguir:

$$
\begin{gathered}
\mathrm{Y}_{\mathrm{ijkml}}=\mu+\alpha_{\mathrm{i}}+\gamma_{\mathrm{j}}+\lambda_{\mathrm{k}}+\beta_{\mathrm{m}}+\alpha \beta_{\mathrm{im}}+\alpha \gamma_{\mathrm{ij}}+\alpha \lambda_{\mathrm{ijk}}+\beta \lambda_{\mathrm{mk}}+\beta \gamma_{\mathrm{mj}}+\gamma \lambda_{\mathrm{jk}}+\alpha \beta \gamma_{\mathrm{imj}} \\
+\alpha \beta \lambda_{\mathrm{imk}}+\alpha \gamma \lambda_{\mathrm{ijk}}+\beta \gamma \lambda_{\mathrm{mjk}}+\alpha \beta \gamma \lambda_{\mathrm{m} \mathrm{mjk}}+\epsilon_{\mathrm{ijkml}}
\end{gathered}
$$

Em que $Y_{i j k m l}$ é o valor observado da variável resposta para a dimensão $i$, distância $j$, Eigenface $k$, valores do fator $k$-vizinhos $m$, e repetição $l ; \mu$ é a média estimada da variável resposta, considerando todas as observações; $\alpha_{i}$ é o efeito estimado da dimensão $i$ na saída; $\beta_{m}$ é o efeito estimado dos valores de $k$-vizinhos $m$ na saída; $y_{j}$ é o efeito estimado dos valores das distâncias $j$ na saída; $\lambda_{k}$ é o efeito estimado dos valores de Eigenfaces $k$ na saída; $\alpha \beta_{i m}$ é a interação entre os fatores primários; $\alpha y_{i j}$ é a interação entre os fatores primários; $\alpha \lambda_{i k}$ é a interação entre os fatores primários; $\beta \lambda_{m k}$ é a interação entre os fatores primários; $\beta y_{m j}$ é a interação entre os fatores primários; $y \lambda_{j k}$ é a interação entre os fatores primários; $\alpha \beta y_{i m j}$ é a interação entre os fatores primários; $\alpha y \lambda_{i j k}$ é a interação entre os fatores primários; $\beta y \lambda_{m j k}$ é a interação entre os fatores primários; $\alpha \beta y \lambda_{i m j k}$ é a interação entre os fatores primários; $\varepsilon_{i j}$ é o erro experimental para a dimensão $i$, distância $j$, Eigenface $k$, valores do fator $k$ vizinhos $m$, e repetição $l$.

$$
\begin{gathered}
\mathrm{Y}_{\mathrm{ijkml}}=0.94+\alpha_{\mathrm{i}}+\gamma_{\mathrm{j}}+\lambda_{\mathrm{k}}+\beta_{\mathrm{m}}+\alpha \beta_{\mathrm{im}}+\alpha \gamma_{\mathrm{ij}}+\alpha \lambda_{\mathrm{ik}}+\beta \lambda_{\mathrm{mk}}+\beta \gamma_{\mathrm{mj}}+\gamma \lambda_{\mathrm{jk}}+\alpha \beta \gamma_{\mathrm{imj}} \\
+\beta \gamma \lambda_{\mathrm{mijk}}+\alpha \beta \gamma \lambda_{\mathrm{m} \mathrm{mk}}+\epsilon_{\mathrm{ijkml}}
\end{gathered}
$$

Tabela 4 - Alocação de variação do experimento

\begin{tabular}{cccc}
\hline Efeito & Alocação de variação & Efeito & Alocação de variação \\
\hline$\alpha$ & $S S \alpha=1,48 \%$ & $\beta \gamma$ & $S S \beta \gamma=2,48 \%$ \\
$\beta$ & $S S \beta=56,3 \%$ & $\beta \lambda$ & $S S \beta \lambda=0,1 \%$ \\
$\gamma$ & $S S \gamma=6,2 \%$ & $\gamma \lambda$ & $S S \gamma \lambda=0,148 \%$ \\
$\lambda$ & $S S \lambda=0,56 \%$ & $\alpha \beta \gamma$ & $S S \alpha \beta \gamma=0,087 \%$ \\
$\alpha \beta$ & $S S \alpha \beta=0,06 \%$ & $\beta \gamma \lambda$ & $S S \beta \gamma \lambda=0,0538 \%$ \\
$\alpha \gamma$ & $S S \alpha \gamma=0,9 \%$ & $\alpha \beta \gamma \lambda$ & $S S \alpha \beta \gamma \lambda=0,0538 \%$ \\
$\alpha \lambda$ & $S S \alpha \lambda=0,114 \%$ & $\epsilon$ & $S S \epsilon=31,44 \%$ \\
\hline \multicolumn{4}{r}{ Legenda: $\alpha=$ dimensão; $\beta=k$-vizinhos; $y=$ distância; $\lambda=$ Eigenfaces; $\varepsilon=$ efeito do erro experimental. } \\
Fonte: elaboração dos autores com base nos dados da pesquisa.
\end{tabular}

Conforme análise dos dados descritos na Tabela 4, o modelo resultante para acurácia está representado na Equação (9). Verificou-se que apenas os efeitos das interações entre os fatores dimensão, distância e Eigenface ( $\alpha y \lambda i j k)$ e entre os fatores dimensão, k e Eigenfaces ( $\alpha \beta \lambda i m k)$ não são significantes. As restantes interações e os fatores principais são significantes. Dessa forma, a fim de simplificar o modelo da acurácia, a Equação (9)

\footnotetext{
${ }^{4}$ Disponível em: <https://docs.google.com/file/d/0B4d9yu3dP4GJdU5GNUY1S2R5ak0/edit>.
} 
somente conteve os respectivos coeficientes e interações entre os fatores. O valor 0.94 é a média estimada do modelo.

\subsection{Validação do modelo}

Uma vez que o modelo matemático da métrica acurácia é identificado, precisa-se validar o modelo identificado da acurácia para poder realizar o teste estatístico corretamente. Após a validação, foram testadas estatisticamente as hipóteses do experimento utilizando-se o método Analysis of variance (Anova) [12].

O modelo representado na Equação (2) foi avaliado por uma análise residual. Essa análise é pré-requisito do teste Anova. A validação do modelo para variável-resposta acurácia foi feita analisando-se as suposições de normalidade, independência dos erros e variação constante dos resíduos, ilustradas na Figura 1.

Figura 1: (A) premissas normalidade, (B) variação para teste Anova da variável acurácia e (C) verificação da premissa independência de erros para teste Anova da variável acurácia

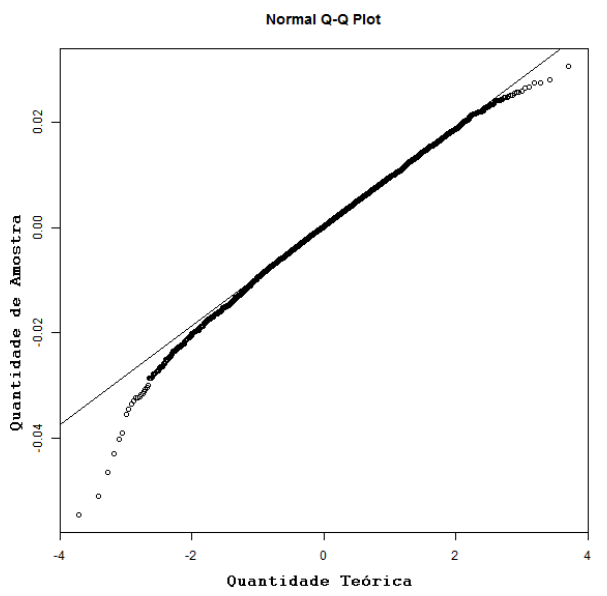

(A)

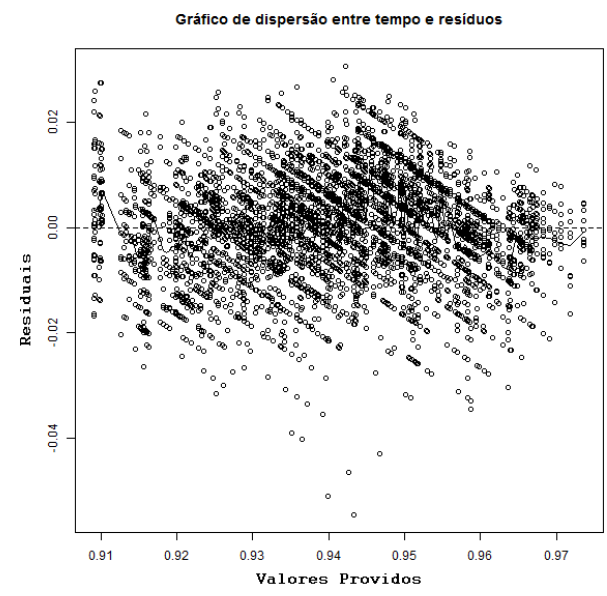

(B)

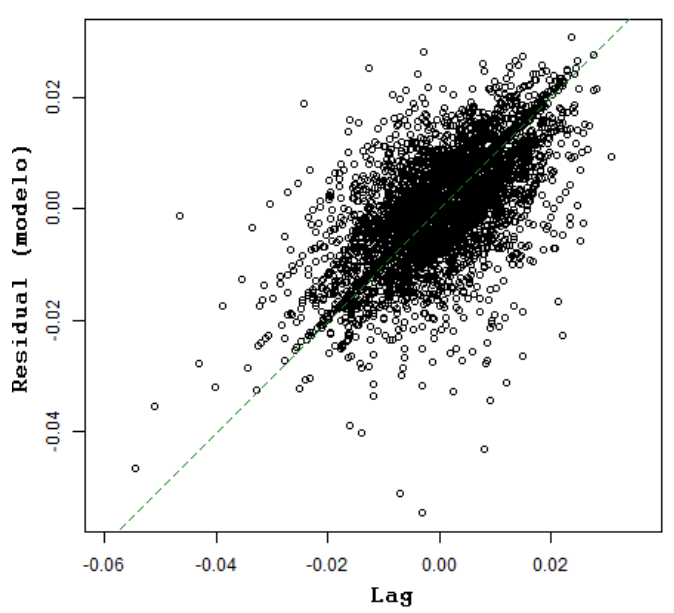

(C)

a) Normalidade: o $Q-Q$ Plot (Figura 1A) sugere que os resíduos não são exatamente normais, possivelmente pela presença de outliers existentes.

b) Independência dos erros: o Lag Plot (Figura 1C) indica que não há forte correlação entre os erros, o que sugere independência dos resíduos.

c) Variação constante: no gráfico de dispersão entre resíduos (Figura 1B), não é possível identificar padrões de crescimento contínuo ("formato de funil") [12], logo, não há indício de que a variação não é constante. 


\section{$5 \quad$ Análise de variância}

As hipóteses desta investigação científica foram testadas de duas formas: a) utilizando o Anova e b) em testes não paramétricos. Nas subseções seguintes, apresenta-se a alocação da variação da variável-resposta entre os efeitos dos fatores, entre os efeitos da interação dos fatores e os efeitos dos erros. Após apresentar a alocação de variação, verificou-se a significância dos efeitos. E, por fim, foram analisadas as hipóteses de investigação formuladas inicialmente.

\subsection{Alocação de variação}

A alocação de variação ajuda a descobrir o percentual de saída que pode ser alocado nos fatores principais, nas interações entre fatores e nos erros. Logo, pode-se entender as explicações das variações usando os fatores e erros, pois sabe-se qual é o maior responsável pela variação da saída. A Tabela 4 detalha os resultados encontrados da alocação de variação.

Foi mostrado, na Tabela 4, que o fator $k$ é responsável por mais de 55\% da variação. Vale ressaltar que o fator $k$ é mais importante para a variável resposta acurácia, pois o classificador K-NN depende dos valores do fator de $k$ para classificar as imagens. Os fatores dos efeitos $\alpha, \beta, y$ e $\lambda$ (dimensão, $k$, distância e Eigenface, respectivamente) explicam aproximadamente $65 \%$ da variância na variável-resposta acurácia do experimento, sendo assim os fatores de maior importância para a variável resposta acurácia.

\subsection{Significância dos efeitos}

Para cada fator e interação entre os fatores da métrica acurácia do experimento, as seguintes hipóteses foram testadas:

a) H0: não há diferença entre os efeitos dos fatores da variável independente;

b) H1: há diferença entre os efeitos dos fatores da variável independente.

Ao realizar um $F$-test, considerando um nível de significância de 5\%, quase todos os fatores rejeitam a hipótese nula. Assim, pode-se afirmar que a maioria dos fatores tem significância estatística em relação à alteração da métrica acurácia. Somente os efeitos da interação entre os fatores dimensão, distância e Eigenface e entre os fatores dimensão, $k$ e Eigenface não rejeitaram a hipótese nula, pois apresentaram seus valores de $F$ menores que o valor da tabela F. A Tabela 5, apresenta os resultados do F-test dos fatores que rejeitaram a hipótese nula.

Tabela 5: Significância dos efeitos para a variável-reposta acurácia

\begin{tabular}{ccccccccc}
\hline & $\boldsymbol{\alpha}$ & $\boldsymbol{\beta}$ & $\boldsymbol{\gamma}$ & $\boldsymbol{\lambda}$ & $\boldsymbol{\alpha} \boldsymbol{\beta}$ & $\boldsymbol{\alpha} \boldsymbol{\gamma}$ & $\boldsymbol{\alpha} \boldsymbol{\lambda}$ \\
\hline SS & 0.0212 & 0.8370 & 0.0922 & 0.0083 & 0.0009 & 0.0133 & 0.0016 \\
$\mathbf{D F}$ & 1 & 1 & 2 & 1 & 1 & 2 & 1 \\
$\mathbf{M S}$ & 0.022 & 0.837 & 0.046 & 0.008 & 0.0009 & 0,0066 & 0.0016 \\
$\mathbf{F}$ & 227.31 & 8660.67 & 477.445 & 85.948 & 191.015 & 69.107 & 17.152 \\
\hline & $\boldsymbol{\beta} \boldsymbol{\gamma}$ & $\boldsymbol{\beta} \boldsymbol{\lambda}$ & $\boldsymbol{\gamma} \boldsymbol{\lambda}$ & $\boldsymbol{\alpha} \boldsymbol{\beta} \boldsymbol{\gamma}$ & $\boldsymbol{\beta} \boldsymbol{\gamma} \boldsymbol{\lambda}$ & $\boldsymbol{\alpha} \boldsymbol{\beta} \boldsymbol{\gamma} \boldsymbol{\lambda}$ & $\boldsymbol{\epsilon}$ \\
\hline SS & 0.0369 & 0.0014 & 0.0021 & 0.0012 & 0.00073 & 0.0007 & 0.4674 \\
DF & 2 & 1 & 2 & 2 & 2 & 2 & 4836 \\
MS & 0.0184 & 0.0014 & 0.001 & 0.0006 & 0.0003 & 0.0003 & 0.00009 \\
F & 191.01 & 15.182 & 11.268 & 6.475 & 3.954 & 3.914 & - \\
\hline
\end{tabular}

Legenda: $\alpha=$ dimensão; $\beta=k$-vizinhos; $y=$ distância; $\lambda=$ Eigenfaces; $\mathbf{E}=$ efeito do erro experimental; $\mathrm{DF}=$ Degree Freedom; SS = Sum Squares; MS = Mean Squares.

Fonte: elaboração dos autores com base nos dados da pesquisa.

\subsection{Verificação das hipóteses}

Uma vez que foi identificado que todos os principais fatores têm significância estatística na variávelresposta acurácia, foram realizados experimentos para avaliar se a taxa de acurácia é estatisticamente significativa em comparação com os parâmetros de todos os fatores. Assim, será feita a análise da verificação das hipóteses estabelecidas. 
Para responder às hipóteses H1-0, H2-0, H3-0 e H4-0, primeiramente, analisou-se visual e separadamente os intervalos de confiança de cada fator presentes nas imagens (Figuras 2, 3, 4 e 5, e Tabela 6).

Tabela 6: Resultado do F-test

\begin{tabular}{ccccc}
\hline & Dimensão & Distância & $\boldsymbol{k}$ & Eigenface \\
\hline Valor F & 227.31 & 477.445 & 8660.670 & 85.948 \\
Tabela F & 3.9 & 3.05 & 3.9 & 3.9 \\
\hline \multicolumn{5}{c}{ Fonte: elaboração dos autores com base nos dados da pesquisa. }
\end{tabular}

Na Figura 2, observam-se os intervalos de confiança ao se comparar as dimensões 120x90, 32x24 e 12x9. $\mathrm{Na}$ análise da Figura 2, verifica-se que os intervalos de confiança das dimensões 12x9 e 32×24 possuem valores de acurácia maiores do que a dimensão 120x90, pois está acima e não há sobreposição. No entanto, não há como afirmar qual é maior ou menor entre as dimensões $12 \times 9$ e 32×24, pois há sobreposição entre os intervalos. Para se ter certeza de qual é a melhor dimensão, foi feita a análise estatística do F-test, conforme detalhado na Tabela 6 .

Figura 2: Intervalo de confiança do fator dimensão

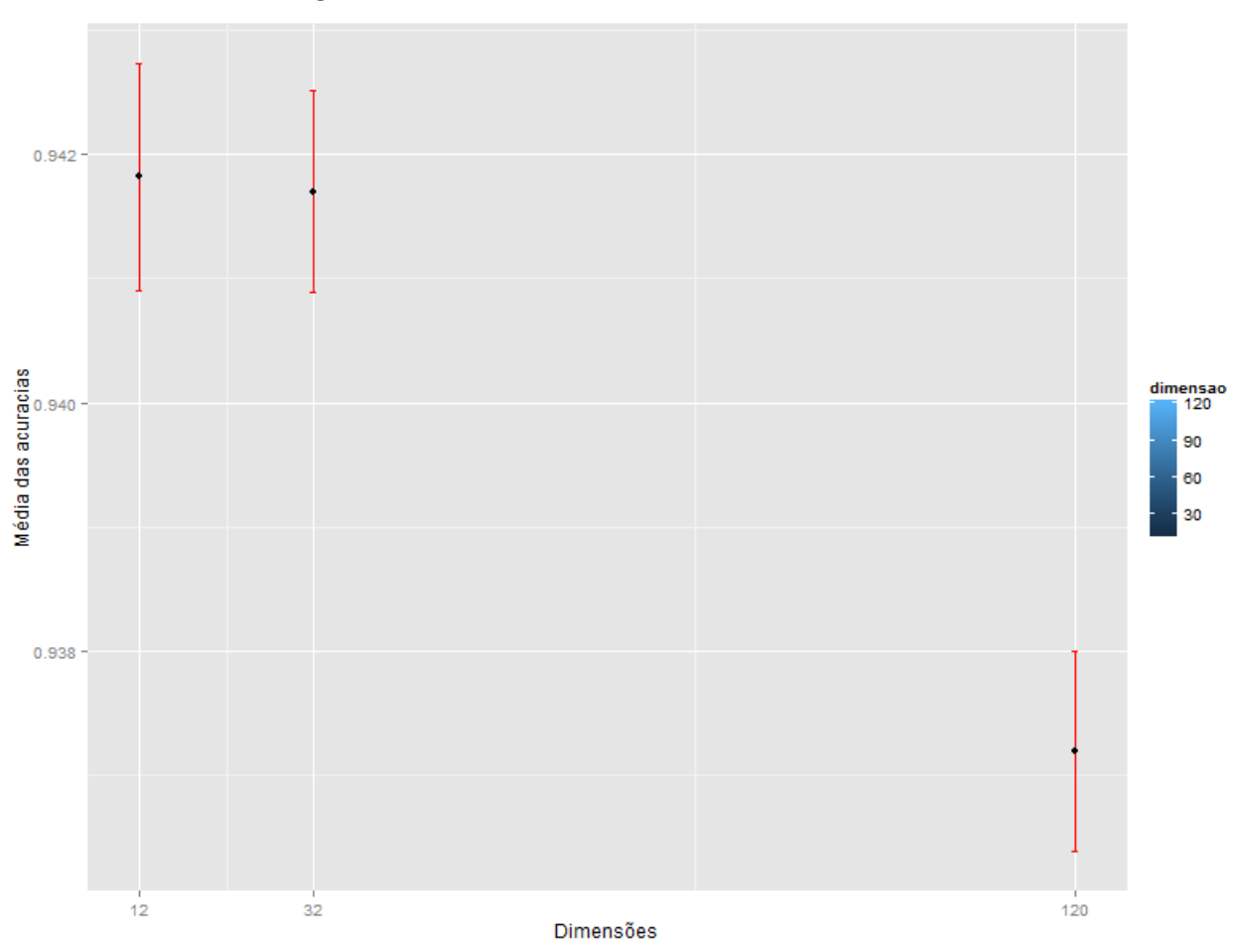

Fonte: elaboração dos autores com base nos dados da pesquisa.

A análise referente aos intervalos de confiança está ilustrada na Figura 3, comparando-se o fator distância aplicado nas imagens com dimensões 120x90, 32×24 e 12x9. Ao analisar a Figura 3, percebe-se que na dimensão $12 \times 9$ a melhor distância é a euclidiana normalizada, seguida da Manhattan e, por último, da euclidiana. Isso para $5 \%$ de nível de significância e independente da realização do F-test. Já nas dimensões 32x24 e 120x90, percebese que a distância euclidiana normalizada tem acurácia maior que as outras distâncias. No entanto, não há como afirmar qual é maior ou menor entre as distâncias Manhattan e euclidiana, pois há sobreposição entre os intervalos. Para se ter certeza de qual é o maior, foi feito um teste de comparação estatístico ( $F$-test), como foi detalhado na Tabela 6. 
Figura 3: Intervalo de confiança do fator distância
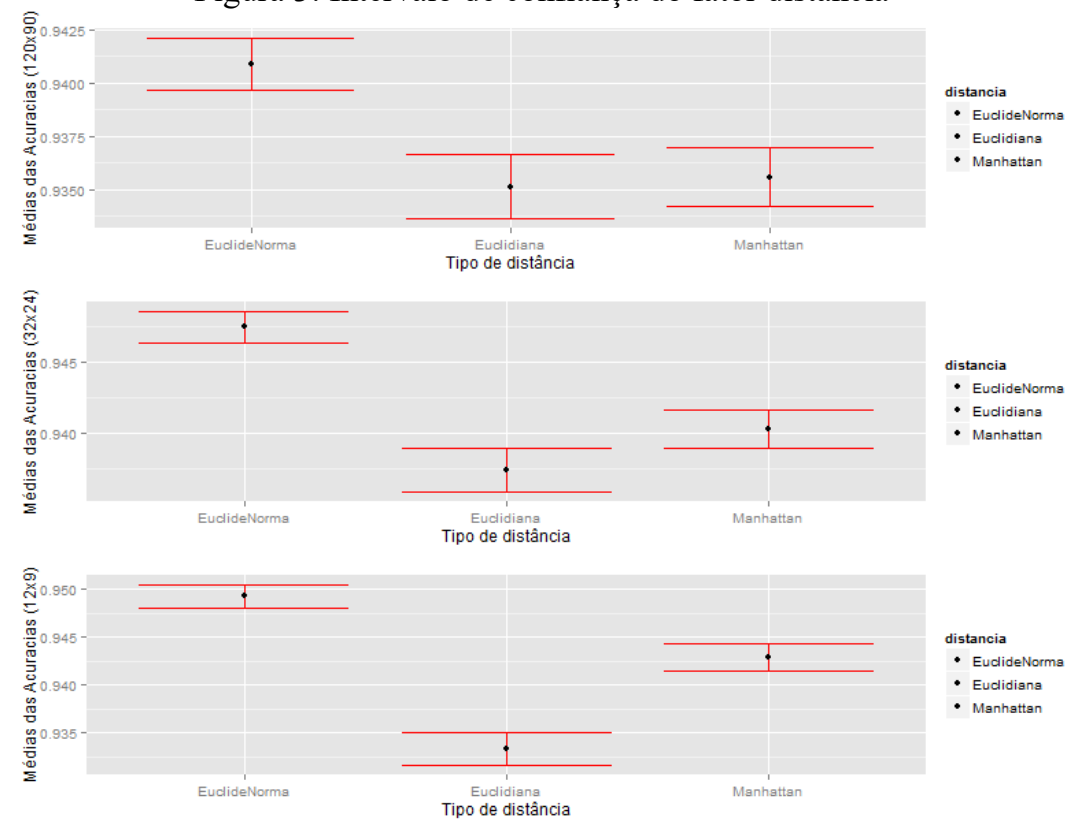

Fonte: elaboração dos autores com base nos dados da pesquisa.

$\mathrm{Na}$ Figura 4, apresenta-se a análise referente aos intervalos de confiança do fator $k$ aplicado nas imagens com dimensões $120 \times 90,32 \times 24$ e $12 \times 9$. Fica evidenciado que, na dimensão $12 \times 9, k=1$ apresenta a maior acurácia, seguida de $\mathrm{k}=4$ e $\mathrm{k}=3$, resultado abordado para $5 \%$ de nível de significância. Evidenciam-se sobreposições entre os fatores $k=5$ e k= 6 e entre $k=9$ e k=10, logo, não há como afirmar qual é maior ou menor entre esses valores. Já nas dimensões 32×24 e 120x90, verifica-se a sobreposição entre k=1 e k=4. Assim, para as dimensões 32x24 e 120x90, constata-se a dificuldade de conhecer qual é o melhor valor $k$ que produz maiores valores para a métrica acurácia. Para se ter a certeza de qual o melhor valor de $k$, foi feito o teste de comparação estatístico $(F$-test $)$, já descrito na Tabela 6.

Figura 4: Intervalo de confiança do fator $k$
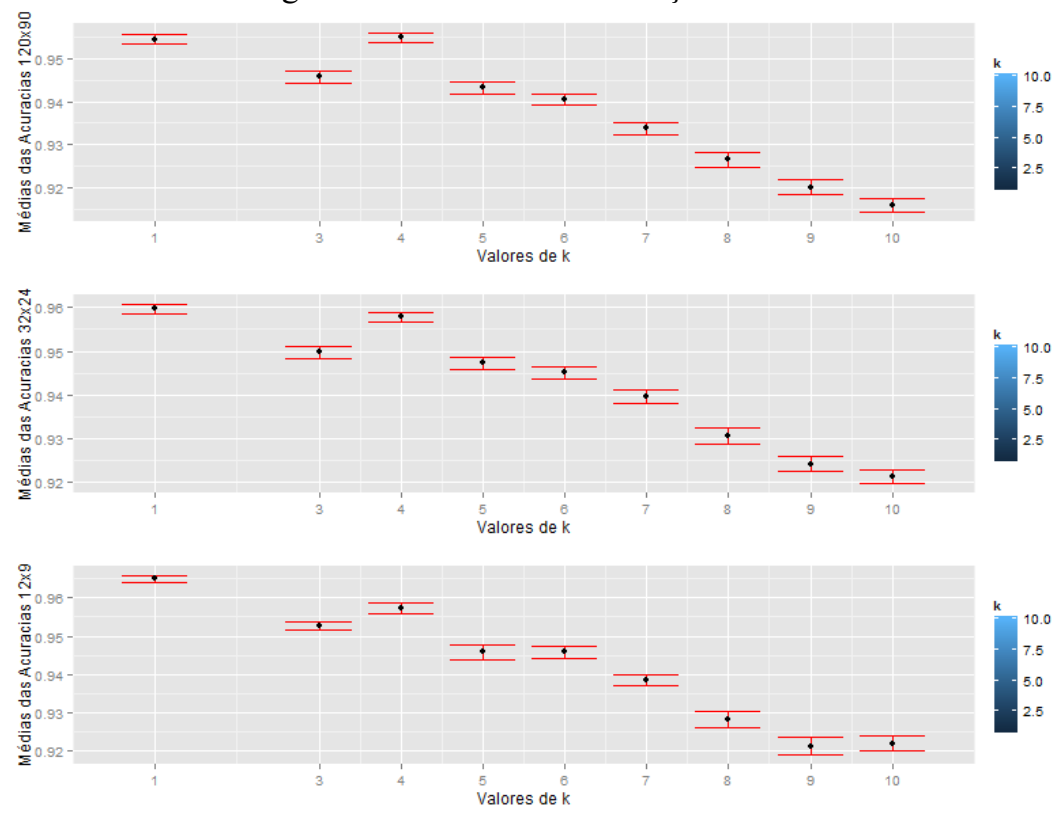

Fonte: elaboração dos autores com base nos dados da pesquisa.

$\mathrm{Na}$ Figura 5, apresenta-se a análise referente aos intervalos de confiança comparando-se o fator Eigenfaces aplicado nas imagens com dimensões 120x90, 32×24 e 12x9. Ao analisar a Figura 5, evidenciam-se 
sobreposições entre os valores de Eigenfaces em todas as dimensões, logo, não há como afirmar qual valor de Eigenface produz maior ou menor valor de acurácia, e se realmente o fator Eigenfaces afeta na acurácia da estimativa do sistema de reconhecimento facial. Para se ter certeza de qual valor Eigenface produz o melhor valor de acurácia, foi feito o teste de comparação estatístico (F-test), cujo resultado também já foi descrito na Tabela 6.
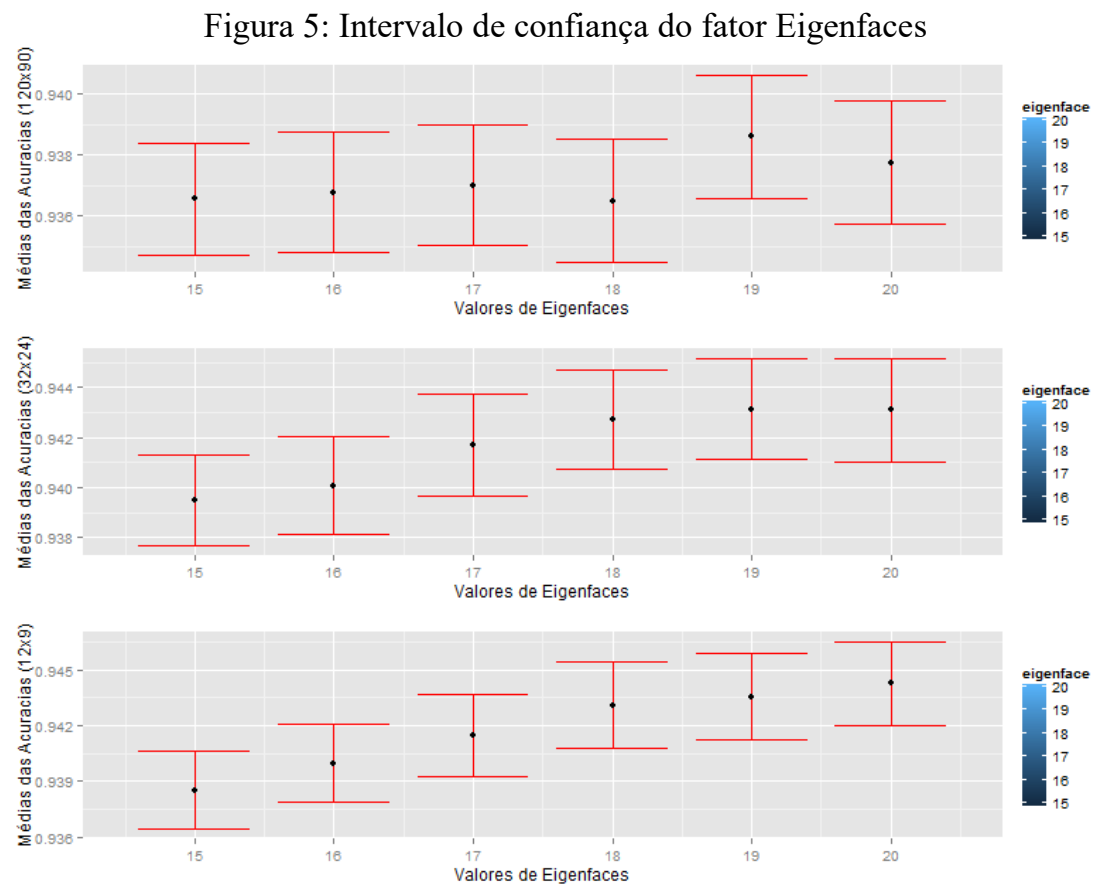

Fonte: elaboração dos autores com base nos dados da pesquisa.

\section{Discussões das verificações das hipóteses}

De acordo com as análises visuais dos intervalos de confiança (Figuras 2, 3, 4 e 5) e dos $F$-tests de todos os fatores abordados (Tabela 6), obteve-se as conclusões que seguem sobre as hipóteses que orientaram este estudo.

Como $\mathbf{F}_{\text {dimensão }}>$ 3.9 implica na rejeição da hipótese H1-0, logo, existe diferença entre os efeitos das dimensões na variável acurácia, e também afeta a acurácia. Pode-se afirmar, analisando-se as informações da Figura 2 e da Tabela 6, que a dimensão 12x9 tem as maiores acurácias. Consequentemente, responde-se à QP1, pois concluiu-se que as dimensões analisadas implicam em que o sistema de reconhecimento facial apresente resultados diferentes na métrica de acurácia, tendo as maiores acurácias resultantes da dimensão $12 \times 9$ e as menores acurácias resultantes da dimensão 120x90.

Como $\mathbf{F}_{\text {distância }}>3.05$ implica na rejeição da hipótese H2-0, logo, o fator distância afeta a variável acurácia. Consequentemente, responde-se à QP2, pois pode-se afirmar, analisando a Figura 3 e a Tabela 6, que a distância euclidiana normalizada é a que produz mais efeito na acurácia com o maior valor, seguida da Manhattan e, por fim, da euclidiana.

Como $\mathbf{F}_{\text {Eigenface }}>$ 3.9 implica na rejeição da hipótese H4-0, logo, o fator Eigenface gera diferentes acurácias. Consequentemente, responde à QP3, pois pode-se afirmar, analisando a Figura 5 e a Tabela 6, que na dimensão 120x90 os melhores Eigenfaces em ordem crescente são: 19, 20, 17, 16, 18, 15. Na dimensão 32x24, os melhores Eigenfaces em ordem crescente são: 19, 20, 18, 17, 16,15. E na dimensão 12x9, os melhores Eigenfaces são: 20, 19, 18,17, 16, 15.

Como $\mathbf{F}_{\mathbf{k}}>3.9$ implica na rejeição da hipótese H3-0, logo, o fator das $k$ afeta a variável acurácia. Consequentemente, responde à $\mathbf{Q P 4}$, pois pode-se afirmar, analisando a Figura 4 e a Tabela 6, que $\mathrm{k}=1$ é o que produziu a maior acurácia, seguido de $\mathrm{k}=4, \mathrm{k}=3, \mathrm{k}=5, \mathrm{k}=6, \mathrm{k}=7, \mathrm{k}=8, \mathrm{k}=9$ e $\mathrm{k}=10$. 
Por fim, concluiu-se que o fator $k$ é que produz os maiores efeitos no resultados da métrica acurácia, seguido dos fatores distância, dimensão e Eigenface respectivamente.

\section{Ameaças à validade do experimento}

Para a avaliação da validade foram considerados dois tipos de avaliações, a externa e a de construção. A validação externa está relacionada com a veracidade aproximada das conclusões e com a generalização da população, já a validade de construção diz respeito às medidas utilizadas.

Com relação à validação externa, uma ameaça que poderá surgir está relacionada à qualidade das imagens da base de dados. Diversos fatores, como baixa luminosidade, contraste ou brilho, podem fazer com que a taxa de acurácia de reconhecimento não seja a mesma observada durante o experimento. Além disso, as unidades experimentais da pesquisa foram selecionadas a partir de uma única fonte (base de dados própria), que pode ter características próprias que não valem para todas as demais bases de dados de imagens faciais. Portanto, há uma ameaça à validade externa na interação de seleção e tratamento, o que dificulta a generalização dos resultados além do escopo estudado.

Por fim, uma possível ameaça à validade de construção relaciona-se aos níveis dos fatores selecionados (quantidade de dimensões das imagens, número de características) que podem não ser suficientes para observar diferenças significativas de eficácia do reconhecimento facial. Desse modo, há uma ameaça à validade de construção causada pela confusão entre constructos e seus níveis.

\section{Conclusões e trabalhos futuros}

As conclusões obtidas após o desenvolvimento deste estudo experimental podem ser divididas em duas dimensões igualmente relevantes. A primeira dimensão é a do processo em si. O método científico e a investigação empírica são realmente indispensáveis para a realização de pesquisa de qualidade. Quanto aos dados coletados, foi realizada uma análise estatística exploratória, direcionada sempre para tentar comprovar ou refutar as hipóteses levantadas sobre o fenômeno em estudo. Foi apresentada uma sequência lógica de como foi realizado o tratamento dos dados, enfatizando-se a importância de cada fator para o modelo e, após a verificação do fator mais importante, se ele realmente é significativo.

Assim, contatou-se que há significância nos efeitos de todos os fatores (distâncias, $k$, Eigenfaces, dimensão) na variável-resposta acurácia, e que o fator $k$ é o mais significativo para explicar a variação nos valores obtidos para a variável de resposta após a execução do sistema. Verificou-se, também, que a dimensão 12x9 produz melhores taxas de acurácias no reconhecimento facial, e que a melhor combinação dos fatores com a melhor acurácia em reconhecimento facial é: dimensão $12 \times 9$, distância euclidiana normalizada, $\mathrm{k}=1$, Eigenface $=20$.

Como propostas de trabalhos futuros, pode-se destacar o uso de novas bases de imagens para realização de mais experimentos assim como a pesquisa de novos fatores que possam influenciar significativamente no reconhecimento de faces com grandes variações de pose e distintas dimensões de imagens. Além disso, devem ser analisados os efeitos dos fatores observados neste trabalho na variável dependente de tempo de processamento.

\section{Agradecimentos}

Os autores agradecem à Capes e ao CNPq pelo suporte parcial a esta pesquisa. 


\section{Referências}

[1] AGARWAL, M. et al. Face recognition using principle component analysis, eigenface and neural network. In: II INTERNATIONAL CONFERENCE ON SENSORS, AND SIGNALS AND VISUALIZATION, IMAGING AND SIMULATION AND MATERIALS, Wisconsin, USA, 204-208, 2009.

[2] ZHAO, W.; CHELLAPPA, R. Face recognition: a literature survey. ACM Computing Surveys, v. 35, n. 4, p. 399-458, 2003.

[3] DINIZ, F. A. et al. RedFace: um sistema de reconhecimento facial baseado em técnicas de análise de componentes principais e autofaces: comparação com diferentes classificadores. Revista Brasileira de Computação Aplicada, Passo Fundo, v. 5, n. 1, p. 42-54, 2013.

[4] KSHIRSAGAR, V. P.; BAVISKAR, M. R.; GAIKWAD, M. E. Face recognition using Eigenfaces. In: III INTERNATIONAL CONFERENCE ON COMPUTER RESEARCH AND DEVELOPMENT, 3, 2011, Shanghai, China 2011. p. 302-306. v. 2.

[5] JIANGSHENG, Y. Method of k-Nearest Neighbors. China - Pequim: Institute of Computational Linguistics, Peking University, 2002.

[6] VIOLA, P. A.; JONES, M. J. Robust real-time object detection. International Journal of Computer Vision, Cambridge, Massachusetts, v. 57, n. 2, p. 137-154, 2004.

[7] CHEON, Y.; KIM, D. A natural facial expression recognition using differential-AAM and K-NNS. Multimedia. In: TENTH IEEE INTERNATIONAL SYMPOSIUM ON MULTIMEDIA, Berkeley, CA, 2008, pp. 220-227.

[8] WOHLIN, C. et al. Experimentation in software engineering: an introduction. Norwell, MA, USA: Kluwer Academic, 2000.

[9] KITCHENHAM, B.; PICKARD, L.; PFLEEGER, S. L. Case studies for method and tool evaluation. IEEE Software, v. 12, n. 4, p. 52-62, 1995.

[10] PRADO, M. P.; SOUZA, S. R. S.; MALDONADO, J. C. Resultados de um estudo de caracterização e avaliação de critérios de teste estruturais entre os paradigmas procedimental e OO. In: X EXPERIMENTAL SOFTWARE ENGINEERING LATIN AMERICAN WORKSHOP, Goiânia - Goiás 2010. p. 91-100.

[11] JURISTO, N.; MORENO A. M. Basics of Software Engineering Experimentation. Boston: Kluver Academic Publischers, 2010.

[12] GRAVETTER, F. J.; WALLNAU, L. B. Statistics for the Behavioral Sciences. Belmont, USA: Cengage Learning, 2013. 